\title{
Study on Chinese Freehand Figure Painting Development Context and Aesthetic Qualities
}

\author{
Rong Ju ${ }^{1}$, Zhaoyang Shao ${ }^{2}$ \\ ${ }^{1,2}$ Agricultural University of Hebei \\ hunter2011@foxmail.com
}

Keywords: Freehand Figure Painting, Freehand Language, Historical Context, Artistic Aesthetics.

\begin{abstract}
Chinese freehand figure painting is an important part of the impressionistic landscapes, an important subcommittee besides bird freehand Chinese painting freehand painting. Figure Drawing formed through a long process of historical development, freehand figure painting also focused on the object by the early perceptual grasp the spiritual content of the development of both traits object and also stressed that the main focus on the performance of emotion, while stressing the ink material charm of its own process.
\end{abstract}

\section{Introduction}

Chinese freehand figure painting is an important part of the impressionistic landscapes, an outside bird freehand Chinese painting freehand painting important subcommittee. Freehand is a unique perceptual in the long process of historical development of painting formed grasp external things and external way of expressing things. Freehand painting is an honor and meticulous work concept for creative methods meticulous in terms of respect.

\section{Chinese Freehand Painting Spirit}

With respect to the Western emphasis on realistic portraits, figure paintings from China on the date of birth has detached object modeling concepts with pictograms above, and freehand figure is a reflection of this concept. On freehand figures, the Italian has three levels, the first level is the object and describe the meaning of the object itself born issued to the "meaning" of specific characteristics of an object including shape, personality traits, as well as the background of his life A comprehensive state cultural background formed. To capture the meaning of the object creators need careful observation and carefully try to figure out. Italian second level of the main creators of art, the art creators because of their personality, learning support, life, world outlook and even emotional state was affected by his view angle and method of external things. Ten thousand books, read a lot of the culture meaning the only way to shape the body. The third level is the main object of a blend of Italy, both "leave on was not the in thing." Broadly speaking excellent works of traditional Chinese painting, whether or freehand brushwork can be said to be the product of the Chinese people to grasp the object of sensibility, are "decent" category, but in this article especially convenient for the narrative proposed a "narrow" the freehand painting concept that freehand figures formed through a long process of historical development, freehand figure painting also focused on the object by the early perceptual grasp the characteristics of the development of the spiritual content of both subject matter and stressed that the main focus on the performance of emotion, while stressing the ink material charm of its own process.

Therefore, freehand painting mature establishes in two separable aspects: one refers to freehand spirit, which the body self-consciousness, art is no longer to imitate and reproduce for the pursuit, began to have a richer spiritual pursuit and individual expression. It is a process by the method of cognition, thus forming a unique method of expression. The second refers to the technique level, which requires pen and ink drawing done both performance and rich Mexican law and Broad strokes can exhibit more spiritual meaning and a step by step to highlight the charm of the material itself. 
Overall, the language of freehand painting has undergone a change and constantly enriches the development of the maturing process. Only simple lines from the early high ancient gossamer later developed to describe the shape of rich and varied depiction 18, further enrich the use of ink changes, eventually forming special painting category and has its own unique artistic language aesthetic process.

\section{Freehand Character Development Context and Aesthetic Characteristics Analysis}

In this article, the author will be divided into the traditional freehand figure gestation period (before the Sui and Tang Dynasties), maturity (the Five Dynasties Song Dynasty), the continuation of the (Yuan and Ming Dynasties), a leap of change (Modern) four times.

Gestation period. The general characteristics of the early period remains in portrait painting, the image of an exaggeration, full of fun, in a linear expression of the main differences between high early ancient gossamer simple, casual stroke of the pen, to mention frustrated by rich variations. Start early accumulation freehand modeling language and on painting techniques.

In each period, for example, depicted on the Qin Dynasty Lian Chu Tomb paint artistic image, the subject matter was of some life style, including travel, wedding marriage, etc. nutshell characters vivid, lively and clever lines. West Hanyang Bu swing tomb of $\mathrm{Fu} \mathrm{Xi}$ image, simple lines, the rate powerful and imaginative. You can feel the obvious lines run body Chi. In addition, the Eastern Han Dynasty tomb murals depicting Balitai door people, pay homage to the scene general officials welcome. Luoyang burning village in historical themes Han story of two peach killing three persons. Exaggerated characters, informal physical things, run lines no longer lingering attachment coiled body structure, but freedom relaxed stretch, showing unrestrained Lvyi mildly rhythm. During this period also shows the national prosperity, vigorous social atmosphere.

Sui and Tang dynasties, freehand figures form factor in the accumulation of skills required to further expand, in terms of the most representative of the line is Huasheng Daozi, his representative works are "King SongZi map", Wu Daozi portrait has a very distinct language features, reflected in the pen, pen fast, bold and imposing, Zhujing Xuan "Tang Dynasty paintings recorded" to describe his pen "scrawled Eqing made there if divine intervention, Shi pen must track, are candid Yat potential" with respect to the Tang period there Liben, Zhang Xuan, Zhou Jin fat rounded fine line language, Daozi lines of language has been significantly change the thickness of the movement, the so-called "Brasenia article," It is a metaphor for this pen. Rich addition to reflect changes in the line of progress beyond the techniques, but also reflects the people more freedom of expression in art.

Thus freehand figure painting language skills required language ready, but regrets that the accumulation of each generate two different families in paintings, one in the field of portrait, one in the field of landscape painting, the two did not form the intersection.

Formation Period (The Five Dynasties Two Song Period). Shi Ke "two ancestral aligning map" depicts two enlightenment monk, a monk tiger pillow and sleep; a monk fall meditation. Jane rate bold ink, and grotesque Peculiar shape, opens up a new realm of Chinese painting, rough freehand pen, ink aspect, character modeling, complete air feet of God, full implication. This change in the subsequent Song of Liang Kai and law often has been further fueled and peaked.

"Patriarch chop bamboo map" of South Song Liang Kai depicting the Sixth Patriarch monk living scene before chop bamboo pen extensive and swift, simple rate character modeling and finish angry enough. Background trees branches dry bulk Feng hastily pen to write, the whole picture crisp Yi-hung, stirring a strong surge of momentum. It is another painting "ink immortal map" can be said Liang Kai minus figure freehand pen style mature work, is freehand figure of the history of large freehand ink representative for depicting a drunk intoxicated intoxicating fairy faltering image, character hand, foot and face few strokes but abundant charm, common drapery lines outline pen sway aspect was replaced by the image become more nutshell, clothes dripping chunk pen, such as pouring majestic.

Continuation Period (Yuan Ming and Qing Dynasties). After the establishment of freehand figures, Yuan, Ming and freehand figure does not like landscapes, flowers and birds as quickly 
theme is accepted, developed, and has been showing a state of stagnation or even ignored, an important reason for the slow development of freehand figures. Traditional figure painting is due to its depiction of a particular object, the main characters, and the characters more flowers in the shape of the landscape is relatively bondage, while painting portraits in functional terms it is more commitment to the "Ming exhort, with ups and downs "of social responsibility. Therefore it is far more sway space landscape flowers much smaller.

More of a portrait depicting literati Gathering, in order to show relaxed, tranquil mood picture on the publicity techniques swift strokes often weakened. Traditional figure painting, the characters topic is rather concentrated religious paintings, since the sample mentoring to teach, from the Yuan Dynasty to the Ming and Qing Dynasties, almost no change on techniques. Freehand figure painter advance breakthrough Song occasion by the ideological influence of Zen Buddhism, but their breakthrough was limited to the historical background has often been ridiculed as "crude evil no healing." For various reasons creating a freehand figure thereafter bird landscape is relatively unbalanced development situation.

Yuan and Ming dynasties freehand figure painting dark Decline, painter is very few. To the Qing Fang, there is little start-up, representative people are Huang Shen, Ren Bo et al.

Revolution Period (Modern). Modern freehand figure to the emergence of a century is a turning point, beginning from the traditional to the modern freehand figure was painted freehand. Such a phenomenon is due to the influence of two resources, a source with the outside culture, along with the Western understanding of different shapes sketch concepts of light and shadow, structure, realistic or performance, greatly enriched the Chinese figure painting freehand modeling concepts and tools. Another breakthrough is derived from the traditional painting of the interior. Traditional pen and ink landscape painting master Huang master of summary that is, its "five-stroke seven ink." Although Mr. Huang Binhong rarely covered portraits, but he re-understanding of modern ink painting freehand figures indicate a new path. It made a breakthrough to ink pen attachment, the idea of the aesthetic characteristics of the ink itself and the further development of connotation.

\section{Conclusion}

Freehand figures is both a traditional painting, but also can be said to be a new kind of painting. Speaking of tradition, its history is long enough, talking about a new respect bird landscape harmony mature, the portrait itself has very few resources to draw on. But because of its new and less, freehand figure painting should be the most development potential and possible draw families. Currently freehand figure showing a flourishing contending situation, in future how the freehand figure to develop the way that will eventually show what kind of look depends on the freehand figure of exploration and every artists.

\section{Acknowledgements}

Project number: K13-08 (This Project is the Key Research Result of Agricultural University of Hebei Curriculum Construction)

Project name: Freehand Figure Painting

\section{References}

[1] Chen-Wen G. Freehand figure Style Talk Concept. Grand Art, 2006, (01).

[2] Y. Wang. Talking about Chinese Describing Figure Drawing. Chinese Art, 2007, (03).

[3] Li-Shao T. Study on Freehand figures Development. Playwright, 2006, (05).

[4] Wang Z. Chinese Realistic Figure Painting "Realism" and "Impressionistic" God-shaped Relationship in Question. Chinese Painters, 2007, (05). 
[5] W. Chen. Chinese Contemporary Freehand Figure Painting Morphology. Journal of Northwest University for Nationalities, 2001, (08). 